A
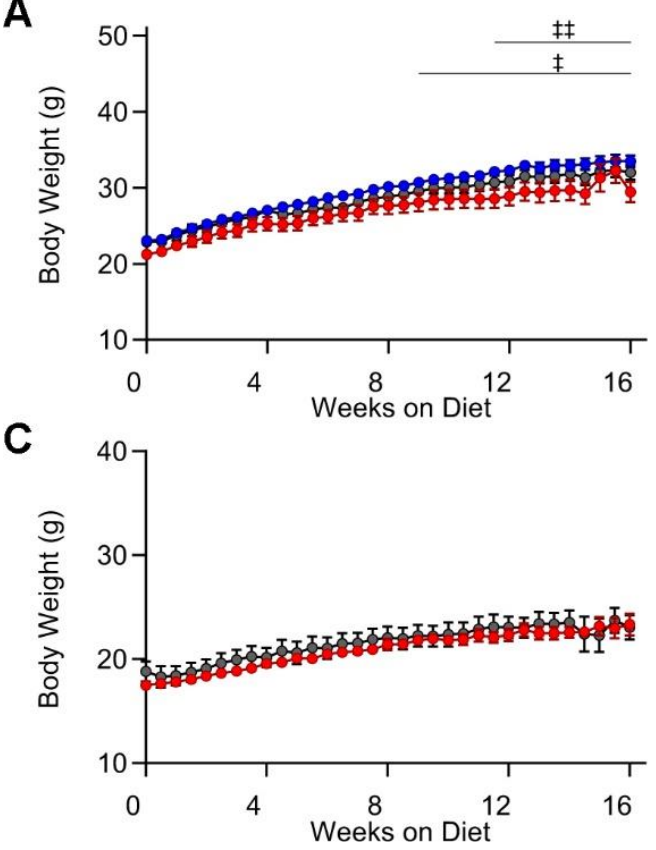
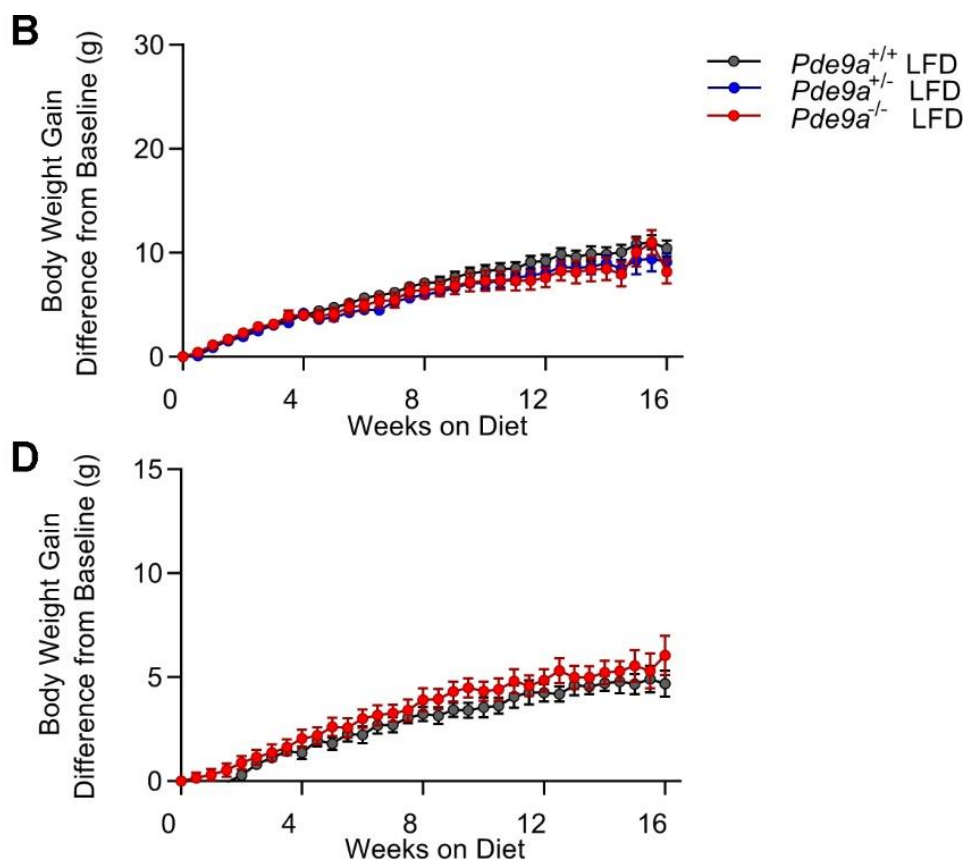

Supplemental Figure S1. Low fat diet fed $P d e 9 a^{-/-}$mice have no difference in body weight.

(A) Male $P d e 9 a^{+/+}, P d e 9 a^{+/-}$, and $P d e 9 a^{-/-}$mice were fed a LFD for 16 weeks beginning at 6 weeks of age. (B) Body weight gain. (C) Female $P d e 9 a^{+/+}$and $P d e 9 a^{-/-}$mice were fed a LFD for 16 weeks beginning at 6 weeks of age. (D) Body weight gain. For (A-B) $\mathrm{N}=10 P d e 9 a^{+/+}$LFD, $17 P d e 9 a^{+/-}$LFD, $11 P d e 9 a^{-/-}$LFD. For (C-D), N = 7 Pde $9 a^{+/+}$LFD, $17 P d e 9 a^{-/-}$LFD. Data were analyzed by 2-way ANOVAs with repeated measures. Post-hoc analyses were performed using Sidak's multiple comparisons test for Pde $9 a$ genotype only and are indicated on figures with $\ddagger$ comparing $P d e 9 a^{+/-}$vs. $P d e 9 a^{-/-}$. ${ }^{*}$ or $\ddagger, \mathrm{P}<0.05 ;{ }^{* *}$ or $\ddagger \ddagger, \mathrm{P}<0.01$. 
A

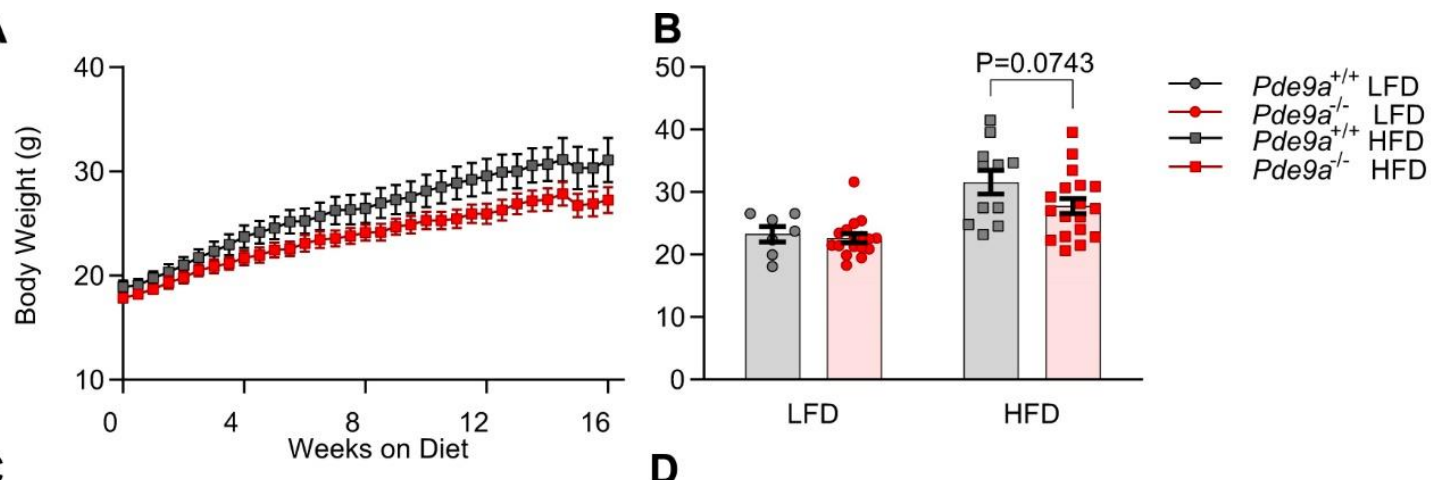

C
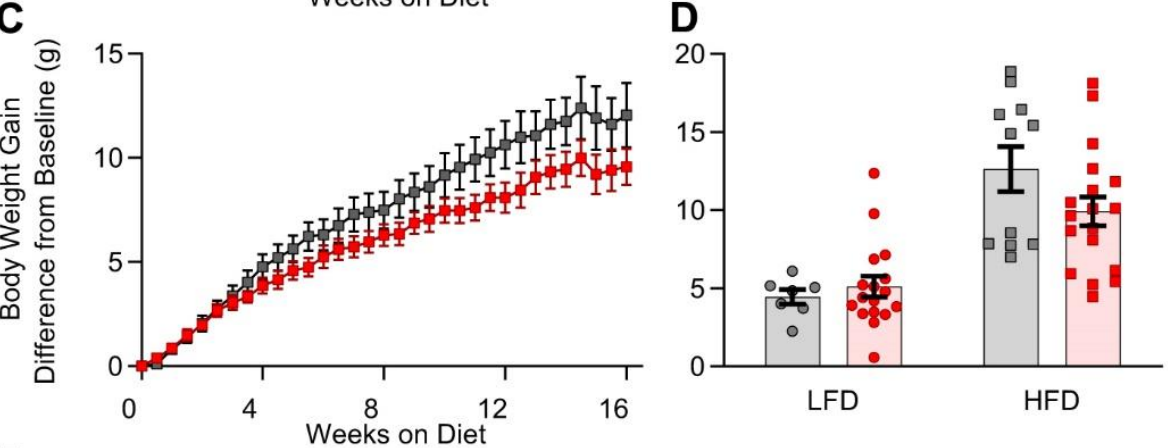

E

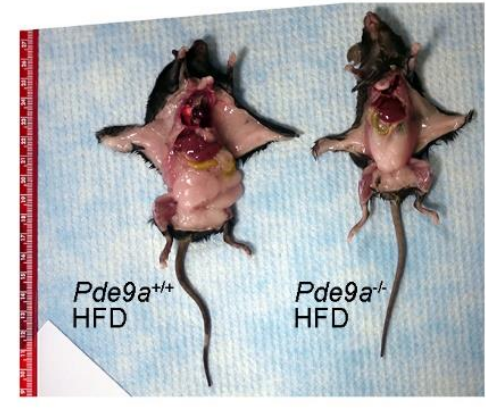

F
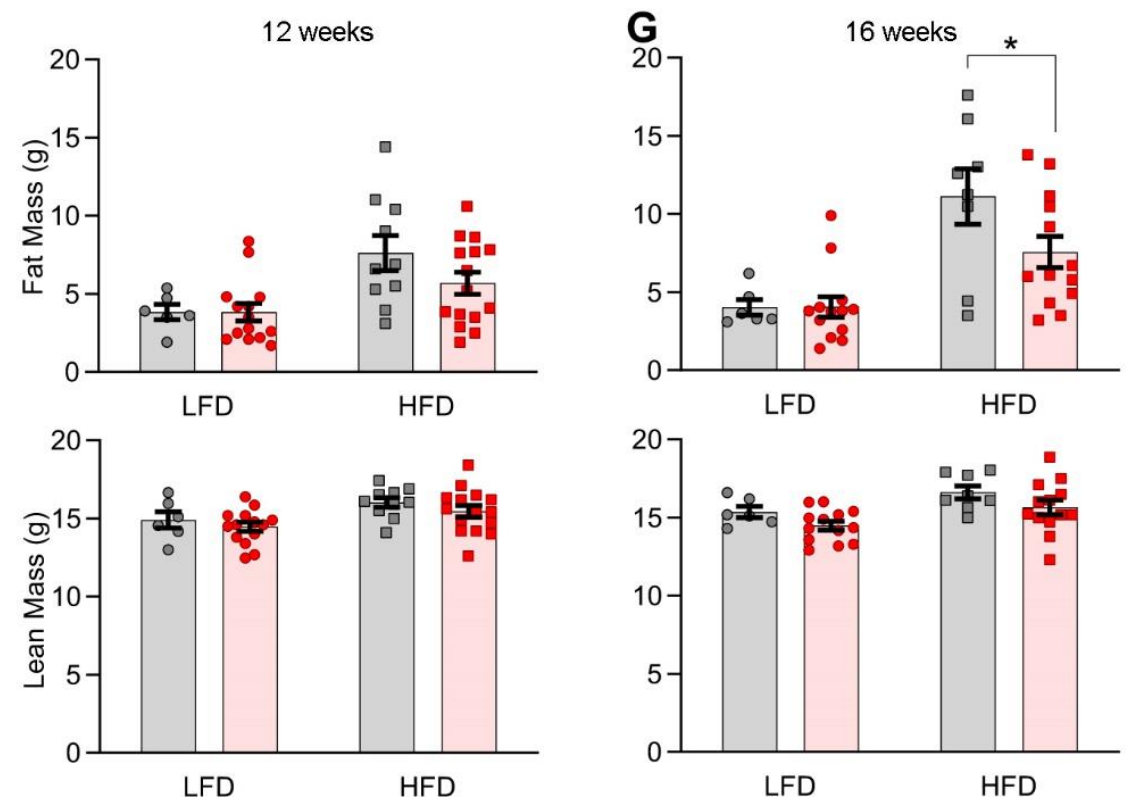
Supplemental Figure S2. Female $P d e 9 a^{-/}$mice exhibit a modest protection from high fat diet induced obesity. (A) Female $P d e 9 a^{+/+}$and $P d e 9 a^{-/-}$mice were fed a LFD or HFD for 16 weeks beginning at 6 weeks of age. LFD results are in Supplemental Figure S1. ( $P=0.0003$, genotype $\times$ time interaction for HFD) (B) Terminal body weight. (C) Body weight gain. LFD results are in Supplemental Figure S1. $(P=0.0003$, genotype $\times$ time interaction for HFD) (D) Cumulative body weight gain. (E) Representative images of Pde $9 a^{+/+}$and $P d e 9 a^{-1-}$ littermates that were fed HFD. Body composition at 12- (F) and 16-weeks (G) of HFD feeding. Data are mean \pm SEM. Analyses were performed using 2-way ANOVA. For (A) and (C), 2-way ANOVAs were performed with repeated measures. Post-hoc analyses were performed using Sidak's multiple comparisons test for Pde $9 a$ genotype only and are indicated on figures with ${ }^{*} \mathrm{P}<0.05$ comparing $P d e 9 a^{+/+}$vs. $P d e 9 a^{-/ /}$. For (A-D), $\mathrm{N}=7$

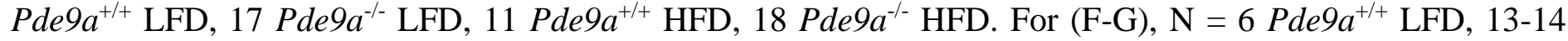
Pde9 $a^{-/-}$LFD, 8-10 Pde9 ${ }^{+/+}$HFD, 13-15 Pde9 $a^{-/-}$HFD. 


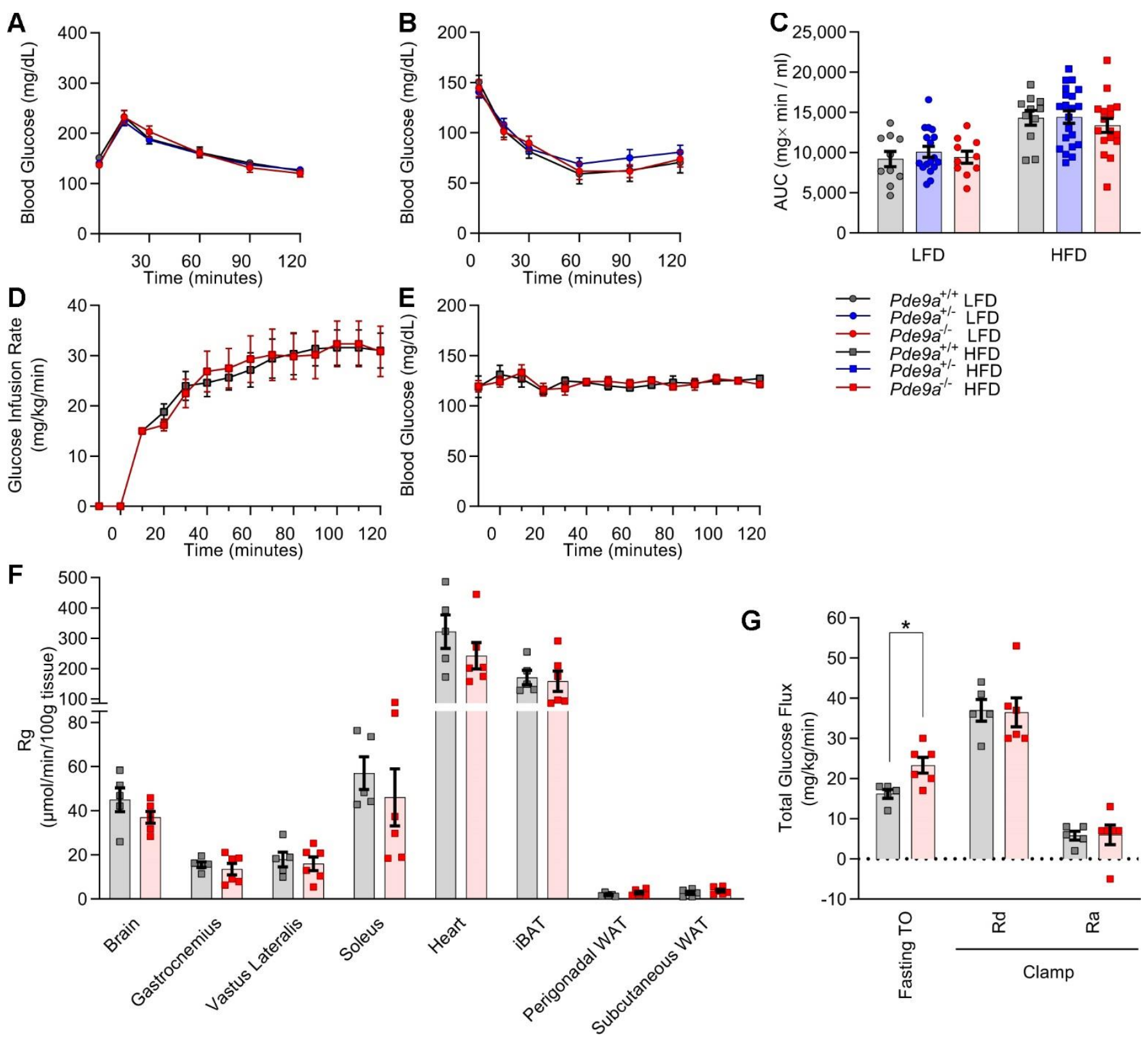

Supplemental Figure S3. Hyperinsulinemic euglycemic clamps revealed no differences in insulin sensitivity between weight matched $P d e 9 a^{+/+}$and $P d e 9 a^{-/-}$mice.

(A) IP-GTT in male $P d e 9 a^{+/+}, P d e 9 a^{+/-}$, and $P d e 9 a^{-/-}$mice fed LFD for 15 weeks. (B) IP-ITT in male $P d e 9 a^{+/+}$, $P d e 9 a^{+/-}$, and $P d e 9 a^{-/-}$mice fed LFD for 14 weeks. (C) Area under the curve (AUC) of ITT data in Figure 3C and Supplemental Figure 2B. (D) Glucose infusion rate. (E) Blood glucose during the clamp. (F) Tissue $\left[{ }^{14} \mathrm{C}\right] 2-\mathrm{deoxy}-$ D-glucose uptake (Rg). (G) Fasting glucose turnover rate in $P d e 9 a^{-/-}$mice compared to $P d e 9 a^{+/+}(\mathrm{P}=0.015)$. Glucose disappearance $(\mathrm{Rd})$ and endogenous glucose production $(\mathrm{Ra})$ during the clamp. Data are mean $\pm \mathrm{SEM}$. For (A) and (B), analyses was performed using 2-way ANOVA with repeated measures. Post-hoc analyses were performed using Sidak's multiple comparisons test. C and D were analyzed by multiple t-tests with statistical significance determined by the Holm-Sidak method with ${ }^{*} \mathrm{P}<0.05 . \mathrm{N}=5 \mathrm{Pde} \mathrm{a}^{+/+} \mathrm{HFD}, 6 \mathrm{Pde}^{-/-} \mathrm{HFD}^{-}$. 

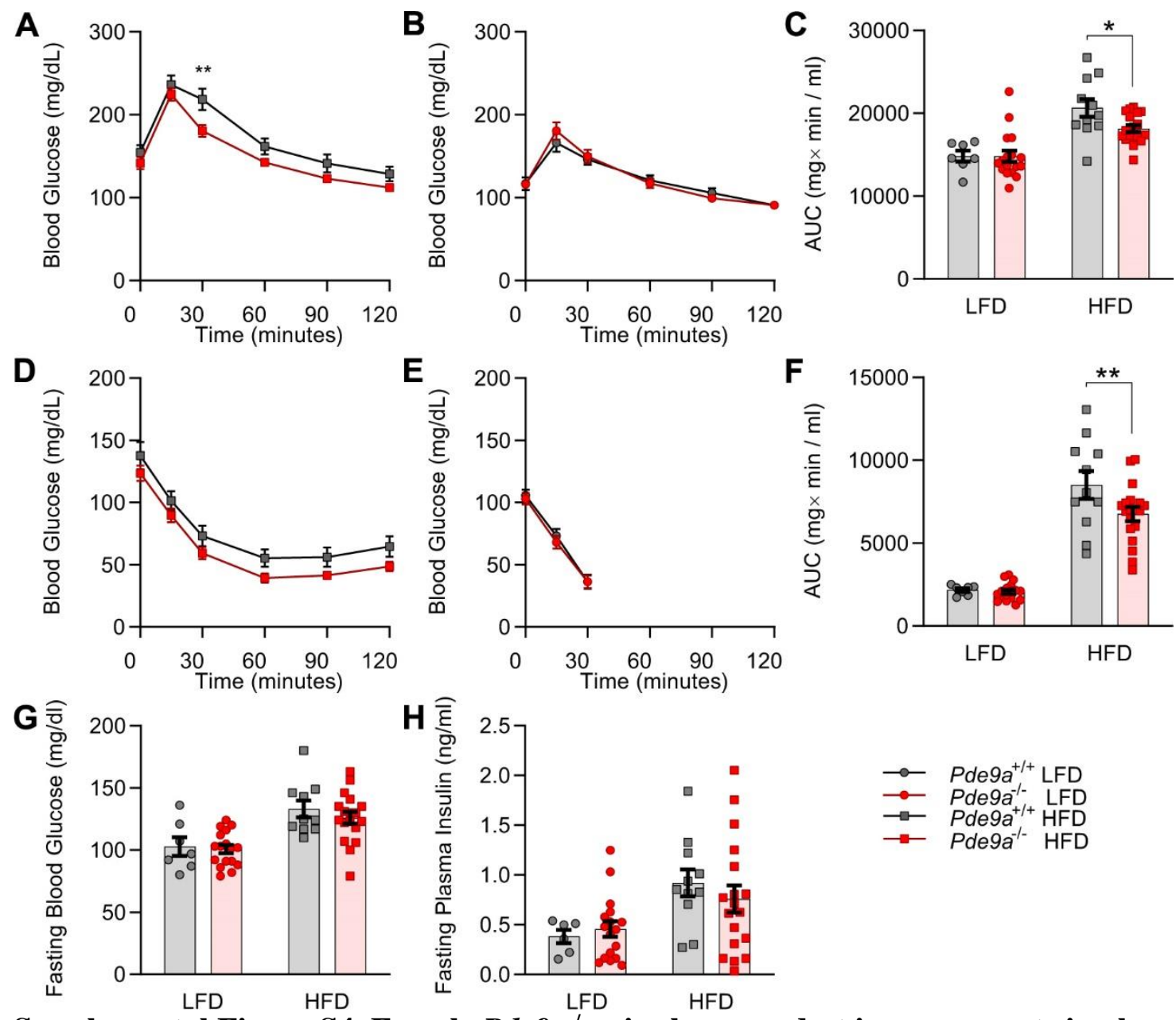

Supplemental Figure S4. Female Pde $9 a^{-/-}$mice have modest improvements in glucose homeostasis.

IP-GTT in female $P d e 9 a^{+/+}$and $P d e 9 a^{-/-}$mice fed either HFD (A) (P = 0.022 effect of genotype) or LFD (B) for 15 weeks. (C) Area under the curve (AUC) of data in (A) and (B). IP-ITT in female $P d e 9 a^{+/+}$and $P d e 9 a^{-/-}$mice fed either HFD (D) (P = 0.059 effect of genotype) or LFD (E) for 14 weeks. (F) Area under the curve (AUC) of data in (D) and (E). Five-hour fasting $(\mathrm{G})$ glucose and $(\mathrm{H})$ insulin at the end of the study. Data are mean \pm SEM. For (A, B, D, and E), 2-way ANOVAs were performed with repeated measures. For (C, G, and H), analyzed by 2-way ANOVA. (F) was analyzed by multiple t-tests. Post-hoc analyses were performed using Sidak's multiple comparisons test for $P d e 9 a$ genotype only and are indicated on figures with ${ }^{*} \mathrm{P}<0.05,{ }^{* *} \mathrm{P}<0.01$ comparing $P d e 9 a^{+/+}$vs. $P d e 9 a^{-/-}$. N $=7 P d e 9 a^{+/+}$LFD, $17 P d e 9 a^{-/-}$LFD, $11 P d e 9 a^{+/+}$HFD, $18 P d e 9 a^{-/-}$HFD. 

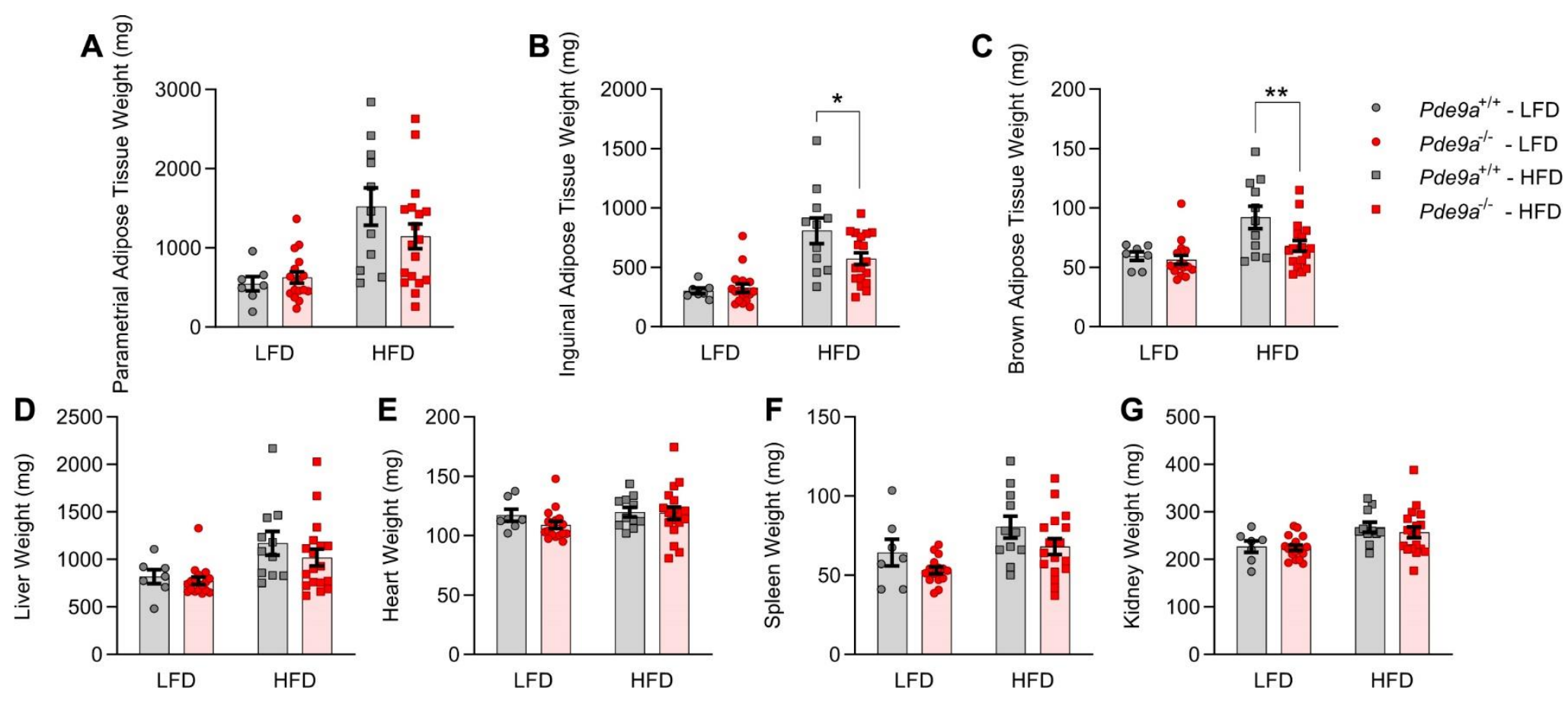

Supplemental Figure S5. Pde9 ${ }^{-/-}$female mice have reduce inguinal and brown adipose tissue weight.

(A) Parametrial WAT weights. (B) iWAT weights $(\mathrm{P}=0.052$, effect of genotypexdiet interaction). (C) iBAT weights $(P=0.030$, effect of genotype). (D) Liver weights. (E) Heart weights. (F) Spleen weights $(P=0.040$, effect of genotype). (G) Kidney weights. Data are mean \pm SEM. Analyses were performed using 2-way ANOVA. Post-hoc analyses were performed using Sidak's multiple comparisons test for Pde $9 a$ genotype only and are indicated on figures with ${ }^{*} \mathrm{P}<0.05,{ }^{* *} \mathrm{P}<0.01$ comparing $P d e 9 a^{+/+}$vs. Pde $9 a^{-/-} . \mathrm{N}=7$ Pde $a^{+/+}$LFD, 17

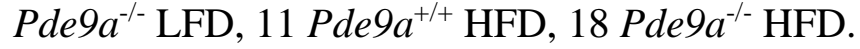



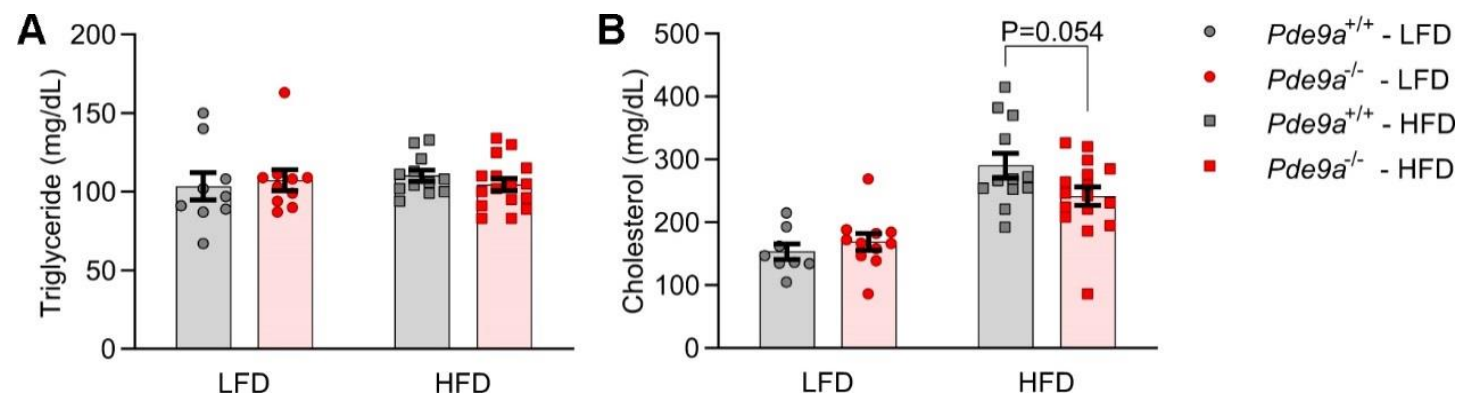

Supplemental Figure S6. Effect of PDE9 on circulating plasma lipids in Pde9a ${ }^{+/+}$and Pde9a mice.

(A) Triglyceride concentrations in plasma from 5-hour fasted mice. (B) Cholesterol concentrations in plasma from 5-hour fasted mice. HFD fed $P d e 9 a^{+/+} v s$. $P d e 9 a^{-/-}$mice $(\mathrm{P}=0.0621$, effect of genotypexdiet interaction). Data are mean \pm SEM. Analyses were performed using 2-way ANOVA. Post-hoc analyses were performed using Sidak's multiple comparisons test for $P d e 9 a$ genotype only and are indicated on figures. $\mathrm{N}=9 \mathrm{Pde} \mathrm{a}^{+/+} \mathrm{LFD}, 12$ Pde $9 a^{-/-}$LFD, $11 P d e 9 a^{+/+}$HFD, $16 P d e 9 a^{-/-}$HFD. 

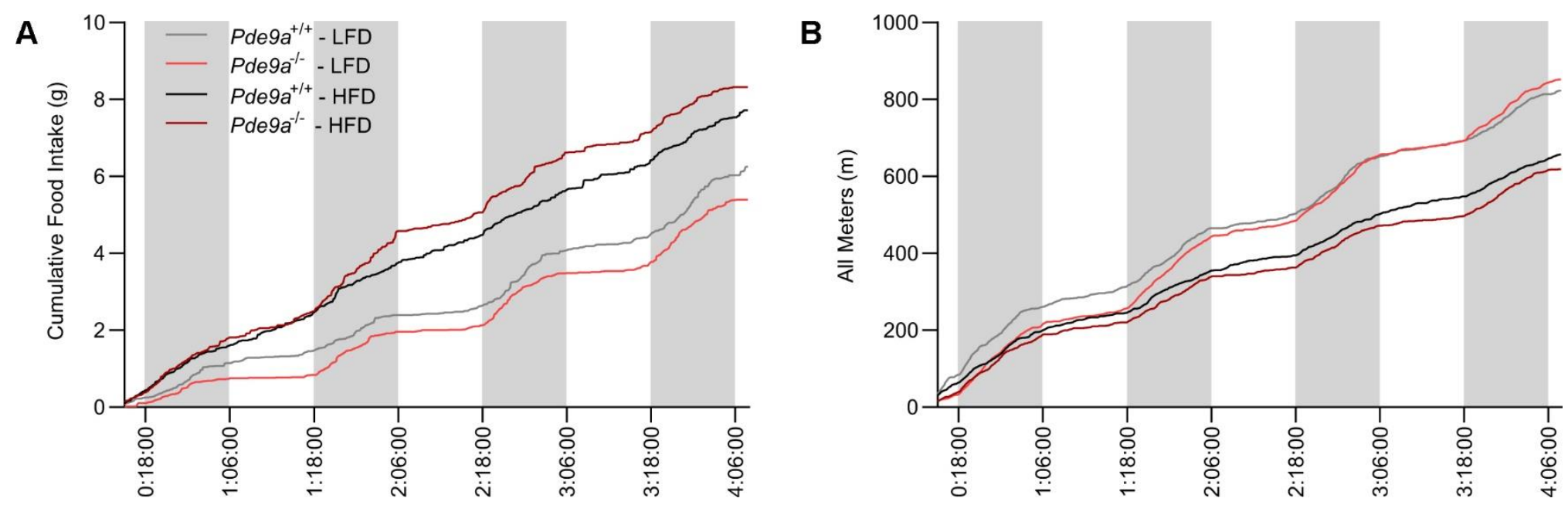

Supplemental Figure S7. Food intake and physical activity.

(A) Cumulative food intake. (B) Cumulative fine and locomotor movement. Values in figure are the mean only from the Promethion System. N = $6 P d e 9 a^{+/+}$LFD, 7 Pde $9 a^{-/-}$LFD, $9 P d e 9 a^{+/+}$HFD, 8 Pde $9 a^{-/-}$HFD. 


$\frac{\text { Control Diet }}{129 a^{+/+}} \frac{P d e 9 a^{-1-}}{123}$
Brown Adipose Tissue
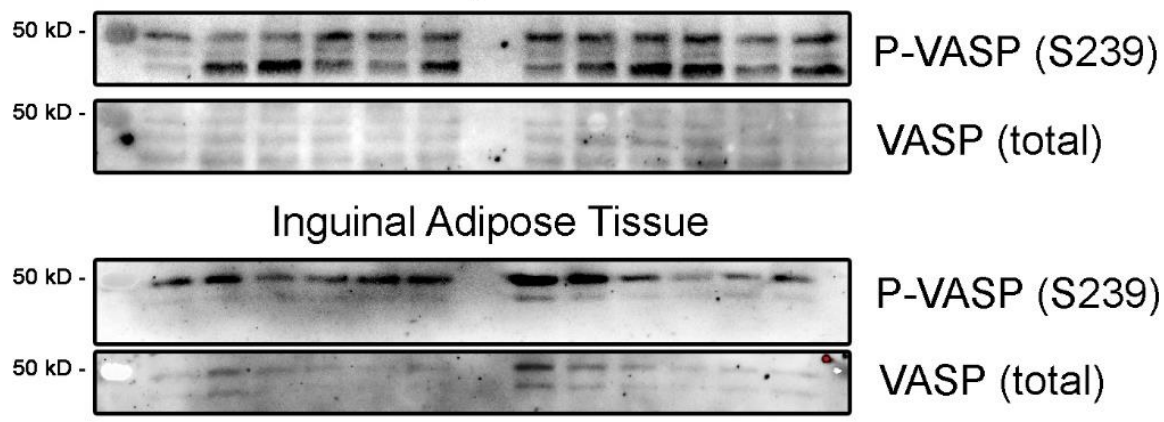

Supplemental Figure S8. VASP ${ }^{\left(\mathbf{S 2 3 9 )}^{2}\right.}$ phosphorylation is not altered under basal conditions P-VASP at $\operatorname{Ser}^{239}$ Western blot from male iBAT and iWAT. 

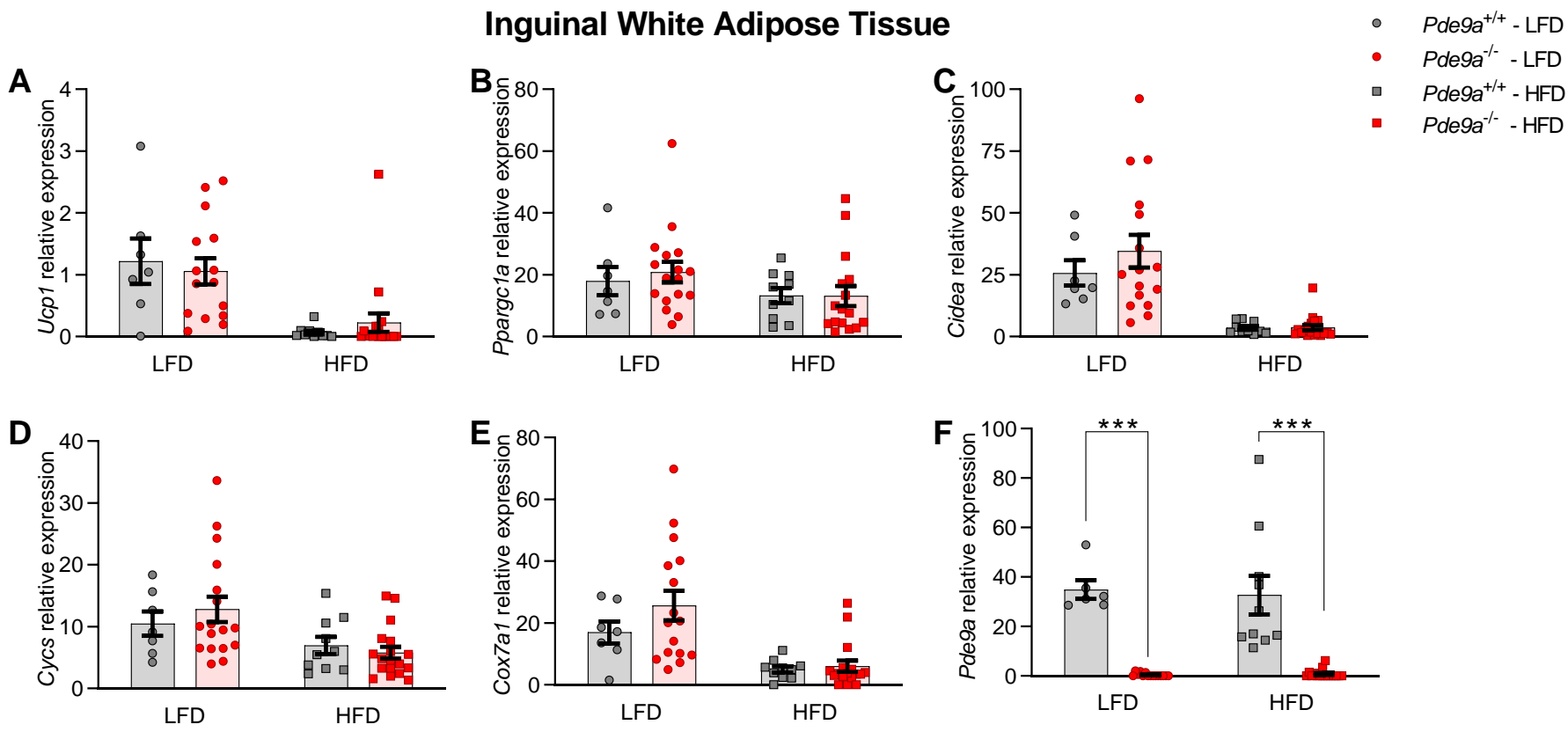

\section{Interscapular Brown Adipose Tissue}
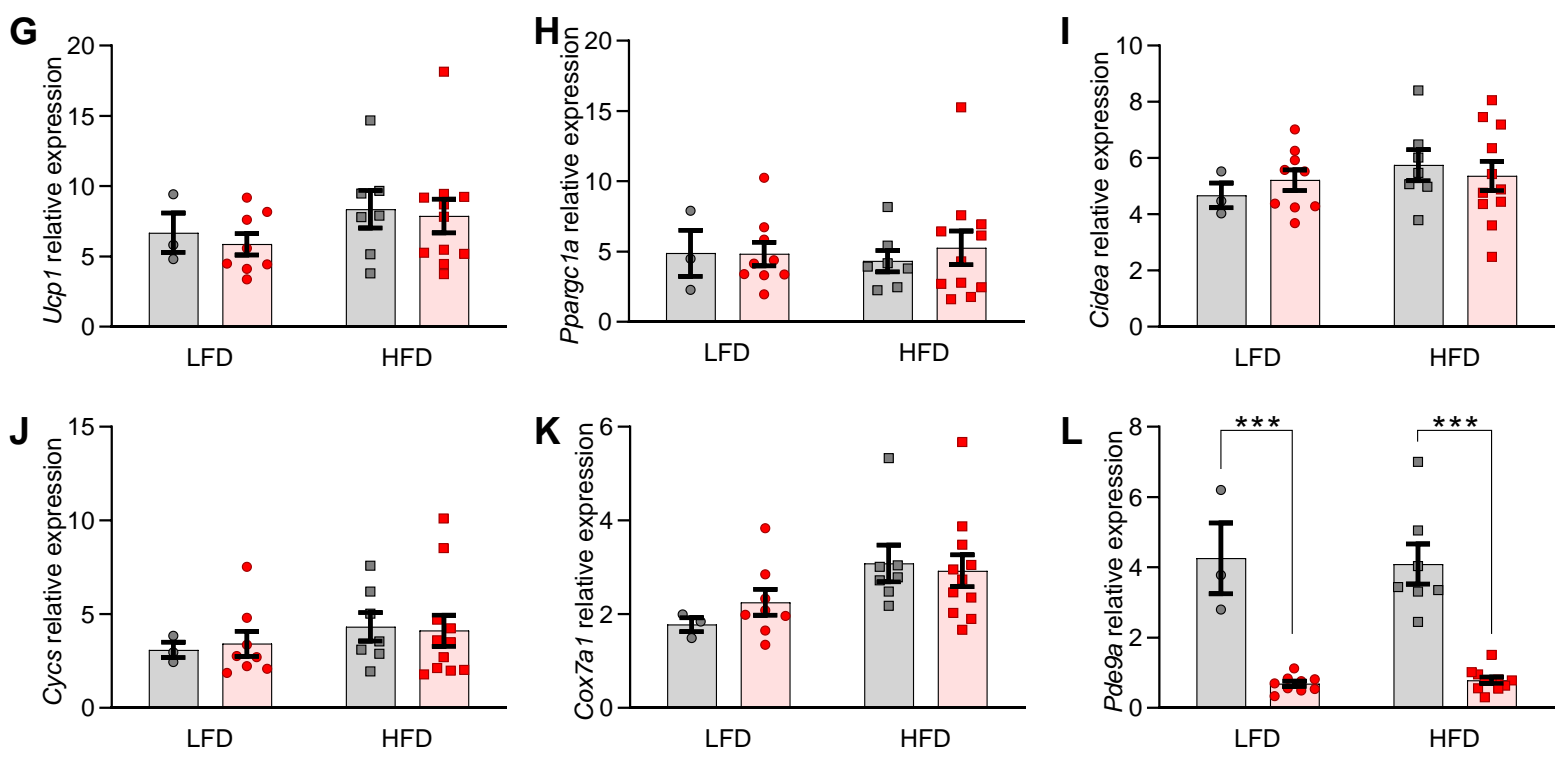

Supplemental Figure S9. Female Pde9 ${ }^{-/-}$mice have unchanged adipose tissue thermogenic gene expression. The iWAT expression of (A) Ucp1 (B) Ppargcla (C) Cidea (D) Cycs (E) Cox7al and (F) Pde9a and the iBAT expression of (G) Ucp1 (H) Ppargcla (I) Cidea (J) Cycs (K) Cox7al and (L) Pde9a by qRT-PCR. Data are mean \pm SEM. Analyses were performed using 2-way ANOVA. Post-hoc analyses were performed using Sidak's multiple comparisons test for $P d e 9 a$ genotype only and are indicated on figures with ${ }^{* * *} \mathrm{P}<0.001$ comparing $P d e 9 a^{+/+}$vs. Pde $9 a^{-/}$. For iWAT, N = 6-7 Pde9 $a^{+/+}$LFD, 15-17 Pde9 $a^{-1-}$ LFD, 10 Pde $9 a^{+/+}$HFD, 16-18 Pde $9 a^{-/-}$HFD. For iBAT, N = 3 Pde $9 a^{+/+}$LFD, 8-9 Pde $9 a^{-/-}$LFD, 7 Pde $9 a^{+/+}$HFD, 11 Pde $9 a^{-/-}$HFD. 


\begin{tabular}{|c|c|c|c|}
\hline Reagent & Final Concentration & $\underline{\text { Source }}$ & Product Code \\
\hline \multicolumn{4}{|l|}{ Chemicals } \\
\hline PF-04447943 & & MedChem Express & HY-15441 \\
\hline ANP (1-28) & & AnaSpec Inc. & AS-20648 \\
\hline BNP & & ProSpec & CYT-369-B \\
\hline BAY 73-6691 & & Sigma & B3561 \\
\hline CL-316,243 & & $\begin{array}{l}\text { American Cyanamid } \\
\text { Co. }\end{array}$ & $\begin{array}{l}\text { gift from Elliott } \\
\text { Danforth Jr. }\end{array}$ \\
\hline Isoproterenol & & Sigma & I6504 \\
\hline pCPT-cGMP & & Sigma & C5438 \\
\hline \multicolumn{4}{|l|}{$\underline{\text { Kits }}$} \\
\hline Mouse Insulin ELISA & & Mercodia & $10-1247-01$ \\
\hline \multicolumn{4}{|l|}{ Cell Culture } \\
\hline \multicolumn{4}{|l|}{ IngJ6 and Bat8 Growth Medium } \\
\hline DMEM/F12 GlutaMAXTM & & ThermoFisher & 10565018 \\
\hline FBS & $15 \%$ & & \\
\hline HEPES & $2 \mathrm{mM}$ & & \\
\hline penicillin and streptomycin & 50 units $/ \mathrm{ml}$ & & \\
\hline \multicolumn{2}{|c|}{ IngJ6 and Bat8 Differentiation Medium } & days $1-4$ & \\
\hline DMEM/F12 GlutaMAXTM & & ThermoFisher & 10565018 \\
\hline FBS & $10 \%$ & & \\
\hline dexamethasone & $5 \mathrm{mM}$ & & \\
\hline Insulin & $0.5 \mathrm{mg} / \mathrm{ml}$ & & \\
\hline isobutylmethylxanthine (IBMX) & $0.5 \mathrm{mM}$ & & \\
\hline rosiglitazone & $1 \mathrm{mM}$ & & \\
\hline T3 & $1 \mathrm{nM}$ & & \\
\hline \multicolumn{2}{|c|}{ IngJ6 and Bat8 Post-differentiation Maintenance Medium } & days $5-8$ & \\
\hline DMEM/F12 GlutaMAX'TM & & ThermoFisher & 10565018 \\
\hline FBS & $10 \%$ & & \\
\hline Insulin & $0.5 \mathrm{mg} / \mathrm{ml}$ & & \\
\hline T3 & $1 \mathrm{nM}$ & & \\
\hline \multicolumn{4}{|l|}{ hMADS Growth Medium } \\
\hline DMEM, low glucose & & Lonza & $12-707 \mathrm{~F}$ \\
\hline FBS & $10 \%$ & & \\
\hline L-glutamine & $2 \mathrm{mM}$ & & \\
\hline HEPES & $10 \mathrm{mM}$ & & \\
\hline
\end{tabular}




\begin{tabular}{|c|c|c|c|}
\hline human FGF-2 & $2.5 \mathrm{ng} / \mathrm{ml}$ & $\begin{array}{l}\text { Shenandoah } \\
\text { Biotechnology }\end{array}$ & $100-146$ \\
\hline penicillin and streptomycin & 50 units $/ \mathrm{ml}$ & & \\
\hline hMADS Differentiation Medium & & days $1-9$ & \\
\hline DMEM/F12 & & Gibco & 11039-021 \\
\hline Dexamethasone & $1 \mu \mathrm{M}$ & & \\
\hline IBMX & $0.5 \mu \mathrm{M}$ & & \\
\hline T3 & $0.2 \mathrm{nM}$ & & \\
\hline insulin & $5 \mu \mathrm{g} / \mathrm{ml}$ & & \\
\hline Rosiglitazone & $1 \mu \mathrm{M}$ & & \\
\hline transferrin & $10 \mu \mathrm{g} / \mathrm{ml}$ & & \\
\hline \multicolumn{2}{|c|}{ hMADS Post-differentiation Maintenance Medium } & days $10-12$ & \\
\hline$\overline{\mathrm{DMEM} / \mathrm{F} 12}$ & & Gibco & 11039-021 \\
\hline T3 & $0.2 \mathrm{nM}$ & & \\
\hline insulin & $5 \mu \mathrm{g} / \mathrm{ml}$ & & \\
\hline Rosiglitazone & $1 \mu \mathrm{M}$ & & \\
\hline transferrin & $10 \mu \mathrm{g} / \mathrm{ml}$ & & \\
\hline \multicolumn{2}{|c|}{ hMADS Post-differentiation Basic Medium } & days $13-16$ & \\
\hline$\overline{\mathrm{DMEM} / \mathrm{F} 12}$ & & Gibco & $11039-021$ \\
\hline insulin & $5 \mu \mathrm{g} / \mathrm{ml}$ & & \\
\hline transferrin & $10 \mu \mathrm{g} / \mathrm{ml}$ & & \\
\hline \multicolumn{4}{|l|}{ Western Blotting Lysis Buffer } \\
\hline HEPES & $25 \mathrm{mM}$ & & \\
\hline $\mathrm{NaCl}$ & $150 \mathrm{mM}$ & & \\
\hline EDTA & $5 \mathrm{mM}$ & & \\
\hline EGTA & $5 \mathrm{mM}$ & & \\
\hline glycerophosphate & $5 \mathrm{mM}$ & & \\
\hline Triton X-100 & $0.9 \%$ & & \\
\hline IGEPAL & $0.1 \%$ & & \\
\hline sodium pyrophosphate & $5 \mathrm{mM}$ & & \\
\hline glycerol & $10 \%$ & & \\
\hline $\begin{array}{l}\text { cOmplete } \\
\text { cocktail }\end{array}$ & $\begin{array}{l}1 \text { tablet/10 ml Lysis } \\
\text { Buffer }\end{array}$ & Roche & 4693124001 \\
\hline PhoSTOP phosphatase inhibitors & $\begin{array}{l}1 \text { tablet/10 ml Lysis } \\
\text { Buffer }\end{array}$ & Roche & 4906845001 \\
\hline \multicolumn{4}{|l|}{ Antibodies } \\
\hline AKT & $1: 1000$ & $\begin{array}{l}\text { Cell Signaling } \\
\text { Technology }\end{array}$ & 9272 \\
\hline
\end{tabular}




\begin{tabular}{|c|c|c|c|}
\hline P-AKT(S473) & $1: 1000$ & $\begin{array}{l}\text { Cell Signaling } \\
\text { Technology }\end{array}$ & 4060 \\
\hline VASP & $1: 1000$ & $\begin{array}{l}\text { Cell Signaling } \\
\text { Technology }\end{array}$ & 3132 \\
\hline P-VASP(S293) & $1: 1000$ & $\begin{array}{l}\text { Cell Signaling } \\
\text { Technology }\end{array}$ & 3114 \\
\hline$\beta$-actin & $1: 2000$ & $\begin{array}{l}\text { Cell Signaling } \\
\text { Technology }\end{array}$ & 4967 \\
\hline UCP1 (Western Blot) & $1: 1000$ & Abcam & $\mathrm{ab} 23841$ \\
\hline UCP1 (IHC) & & Abcam & ab10983 \\
\hline $\begin{array}{l}\text { Goat Anti-Rabbit IgG -Alkaline } \\
\text { Phosphatase }\end{array}$ & $1: 20000$ & MilliporeSigma & A3687 \\
\hline \multicolumn{4}{|c|}{ RNA Purification and Quantitative RT-PCR } \\
\hline Trizol Reagent & & Ambion & 15596018 \\
\hline Zymo-Spin IIICG Column & & Zymo Research & C1006-250-G \\
\hline RNA Prep Buffer & & Zymo Research & R1060-2-100 \\
\hline RNA Wash Buffer & & Zymo Research & R1003-3-48 \\
\hline \multicolumn{2}{|c|}{ High-Capacity cDNA Reverse Transcription Kit } & Applied Biosystems & 4368814 \\
\hline PowerUp SYBR Green Master Mix & & Applied Biosystems & A25742 \\
\hline \multicolumn{4}{|l|}{ GTT and ITT } \\
\hline $0.9 \%$ Saline & & Hospira, Inc. & NDC 0409-7138-09 \\
\hline dextrose $50 \%$ & & Agri Laboratories & NDC 57561-801-50 \\
\hline Insulin (Humulin R) & & Lilly & HI-213 \\
\hline \multicolumn{4}{|l|}{ Oroboros Buffers and Reagents } \\
\hline \multicolumn{4}{|l|}{ Malate 0.4 M } \\
\hline$\overline{\text { Malic Acid }}$ & $400 \mathrm{mM}$ & Sigma & M1000 \\
\hline \multicolumn{4}{|l|}{ ph 7.0 with $\mathrm{KOH}$} \\
\hline \multicolumn{4}{|l|}{ Pyruvate $1 \mathrm{M}$} \\
\hline Sodium Pyruvate & $1 \mathrm{M}$ & Sigma & P2256 \\
\hline \multicolumn{4}{|l|}{ ADP 0.5 M } \\
\hline$\overline{\mathrm{ADP}}$ & $500 \mathrm{mM}$ & Alfa Aesar & L14029 \\
\hline $\mathrm{MgCl}_{2} \bullet 6 \mathrm{H}_{2} \mathrm{O}$ & $300 \mathrm{mM}$ & Fisher & BP214 \\
\hline \multicolumn{4}{|l|}{ ph 7.0 with $\mathrm{KOH}$} \\
\hline \multicolumn{4}{|l|}{ Succinate 1M } \\
\hline Succinic Acid & $1 \mathrm{M}$ & TCI & S0100 \\
\hline ph 7.0 & & & \\
\hline
\end{tabular}




\begin{tabular}{|c|c|c|c|}
\hline \multicolumn{4}{|c|}{$100 \mathrm{mM} \mathrm{K} 2$ EGTA buffer } \\
\hline EGTA & $100 \mathrm{mM}$ & Sigma & E4378 \\
\hline $\mathrm{KOH}$ & $200 \mathrm{mM}$ & Fisher & P250 \\
\hline \multicolumn{4}{|l|}{ ph 7.0 with $\mathrm{KOH}$} \\
\hline \multicolumn{4}{|c|}{$100 \mathrm{mM} \mathrm{CaK}_{2}$ EGTA buffer } \\
\hline $\mathrm{CaCO}_{3}$ & $100 \mathrm{mM}$ & Sigma & 795445 \\
\hline EGTA & $100 \mathrm{mM}$ & Sigma & E4378 \\
\hline $\mathrm{KOH}$ & $200 \mathrm{mM}$ & Fisher & P250 \\
\hline \multicolumn{4}{|l|}{ ph 7.0 with $\mathrm{KOH}$} \\
\hline \multicolumn{4}{|c|}{ Biopsy Preservation Solution: BIOPS } \\
\hline $\mathrm{CaK}_{2}$ EGTA buffer & $2.77 \mathrm{mM}$ & & \\
\hline $\mathrm{K}_{2}$ EGTA buffer & $7.23 \mathrm{mM}$ & & \\
\hline $\mathrm{Na}_{2} \mathrm{ATP}$ & $5.77 \mathrm{mM}$ & Sigma & A2383 \\
\hline $\mathrm{MgCl}_{2} \bullet 6 \mathrm{H}_{2} \mathrm{O}$ & $6.56 \mathrm{mM}$ & Fisher & BP214 \\
\hline Taurine & $20 \mathrm{mM}$ & Sigma & T0625 \\
\hline $\mathrm{Na}_{2} \mathrm{Phosphocreatine}$ & $15 \mathrm{mM}$ & Sigma & P7936 \\
\hline Imidazole & $20 \mathrm{mM}$ & Sigma & 56750 \\
\hline Dithiorthreitol & $0.5 \mathrm{mM}$ & Sigma & D9779 \\
\hline MES hydrate & $50 \mathrm{mM}$ & Sigma & M8250 \\
\hline Fatty Acid Free BSA & $0.1 \%$ & Calbiochem & 126575 \\
\hline \multicolumn{4}{|l|}{ ph 7.1 with $\mathrm{KOH}$} \\
\hline \multicolumn{4}{|l|}{ BIOPS+Saponin } \\
\hline Saponin & $50 \mathrm{ng} / \mathrm{ml}$ in BIOPS & Sigma & S7900 \\
\hline \multicolumn{4}{|c|}{ Mitochondrial Respiration Medium: MiR05 } \\
\hline EGTA & $0.5 \mathrm{mM}$ & Sigma & E4378 \\
\hline $\mathrm{MgCl}_{2} \cdot 6 \mathrm{H}_{2} \mathrm{O}$ & $3.0 \mathrm{mM}$ & Fisher & BP214 \\
\hline Lactobionic acid & $60 \mathrm{mM}$ & RPI & L23000 \\
\hline Taurine & $20 \mathrm{mM}$ & Sigma & T0625 \\
\hline $\mathrm{KH}_{2} \mathrm{PO}_{4}$ & $10 \mathrm{mM}$ & Fisher & $\mathrm{P} 285$ \\
\hline HEPES & $20 \mathrm{mM}$ & Sigma & $\mathrm{H} 4034$ \\
\hline Sucrose & $110 \mathrm{mM}$ & EMD & SX1075-1 \\
\hline Fatty Acid-Free BSA & $0.1 \%$ & Calbiochem & 126575 \\
\hline ph 7.1 with $\mathrm{KOH}$ & & & \\
\hline
\end{tabular}

Supplemental Table S1. List of Materials 


$\begin{array}{cc}\text { Gene } & \text { Forward } \\ \text { mRplpo } & \text { GATGCCAGGGAAGACAG } \\ \text { mCidea } & \text { GTCTGCAAGCAACCAAAGAA } \\ \text { mCox7al } & \text { CGAAGAGGGGAGGTGACTC } \\ m \text { Cycs } & \text { ACCAAATCTCCACGGTCTGTTCGG } \\ \text { mPde5a } & \text { ACAAAGGCATTGTGGGACAT } \\ \text { mPde9a } & \text { AGATGGACATCTTGGTCCTGA } \\ \text { mPpargcla } & \text { CGGAAACATATCCAACCAG } \\ \text { mUcpl } & \text { GGCCTCTACGACTCAGTCCA }\end{array}$

ACAATGAAGCATTTTGGATAATCA
AtTGAGACAGCCGAGGAAGT
AGCCTGGGAGACCCGTAG
GGTGATGCCTTTGTTCTTGTTGGC
TTGGTCAACTTCTGCATTGAA
CGGGCATTGATCTGGTATGT
TGAGAACCGCTAGCAAGTTTG
TAAGCCGGCTGAGATCTTGT

Efficiency

$88.49 \%-105.15 \%$ $101.65 \%-108.65 \%$ $101.2 \%-108.29 \%$ $102.16 \%-103.01 \%$ $98.17 \%-104.14 \%$ $97.04 \%-112.95 \%$ $97.02 \%-107.41 \%$ $93.57 \%-111.9 \%$

Supplemental Table S2. Primer Sequences 


\section{Fatty acid composition of hepatic triglycerides}

\begin{tabular}{|c|c|c|c|c|c|c|c|c|c|c|c|}
\hline Parameter & \multicolumn{2}{|c|}{$\mathrm{Pde} \mathrm{a}^{+/+}-\mathrm{LFD}$} & \multicolumn{2}{|c|}{$P d e 9 a^{-/-}$- LFD } & \multicolumn{2}{|c|}{$P d e 9 a^{+/+}-\mathrm{HFD}$} & \multicolumn{2}{|c|}{ Pde9a ${ }^{-1-}$ - HFD } & \multicolumn{3}{|c|}{ ANOVA p-value } \\
\hline & $\begin{array}{l}\frac{\text { mean }}{g / 100 ~} \mathrm{~g} \\
\text { acids }\end{array}$ & $\underline{\text { SEM }}$ & mean & $\underline{\text { SEM }}$ & mean & $\underline{\text { SEM }}$ & mean & $\underline{\text { SEM }}$ & Genotype & Diet & Interaction \\
\hline $12: 0$ & 0.38 & 0.11 & 0.28 & 0.08 & 2.05 & 0.37 & 2.12 & 0.24 & 0.974 & $<0.001$ & 0.736 \\
\hline $14: 0$ & 1.74 & 0.17 & 1.60 & 0.11 & 5.05 & 0.36 & 5.77 & 0.38 & 0.366 & $<0.001$ & 0.193 \\
\hline $16: 0$ & 25.49 & 0.76 & 27.28 & 0.38 & 31.31 & 0.54 & 31.22 & 0.51 & 0.137 & $<0.001$ & 0.101 \\
\hline $16: 1$ & 8.80 & 0.31 & 8.07 & 0.46 & 7.63 & 0.29 & 7.06 & 0.35 & 0.081 & 0.005 & 0.825 \\
\hline $17: 0$ & 0.00 & 0.00 & 0.00 & 0.00 & 0.00 & 0.00 & 0.00 & 0.00 & NA & NA & NA \\
\hline $18: 0$ & 1.98 & 0.23 & 2.04 & 0.22 & 2.64 & 0.20 & 2.76 & 0.19 & 0.678 & 0.002 & 0.895 \\
\hline $18: 1 \omega 9$ & 42.00 & 0.87 & 42.08 & 0.78 & 36.60 & 0.88 & 35.77 & 0.66 & 0.642 & $<0.001$ & 0.574 \\
\hline $18: 1 \omega 7$ & 7.29 & 0.50 & 7.59 & 0.42 & 5.56 & 0.47 & 5.42 & 0.25 & 0.848 & $<0.001$ & 0.596 \\
\hline $18: 2$ & 10.25 & 0.74 & 9.72 & 0.64 & 6.63 & 0.57 & 7.15 & 0.54 & 0.989 & $<0.001$ & 0.404 \\
\hline $18: 3 \omega 6$ & 0.02 & 0.02 & 0.00 & 0.00 & 0.06 & 0.03 & 0.11 & 0.04 & 0.657 & 0.016 & 0.210 \\
\hline $18: 3 \omega 3$ & 0.49 & 0.04 & 0.30 & 0.06 & 0.27 & 0.04 & 0.25 & 0.07 & 0.083 & 0.038 & 0.184 \\
\hline $20: 3 \omega 6$ & $0.31^{*}$ & 0.06 & $0.12^{*}$ & 0.05 & 0.27 & 0.03 & 0.25 & 0.04 & 0.029 & 0.334 & 0.080 \\
\hline $20: 4$ & 0.66 & 0.08 & 0.50 & 0.11 & 0.71 & 0.11 & 0.79 & 0.09 & 0.716 & 0.107 & 0.237 \\
\hline $20: 5$ & 0.00 & 0.00 & 0.00 & 0.00 & 0.04 & 0.02 & 0.09 & 0.05 & 0.412 & 0.033 & 0.412 \\
\hline $22: 4 \omega 6$ & 0.04 & 0.03 & 0.06 & 0.03 & 0.19 & 0.04 & 0.18 & 0.03 & 0.904 & $<0.001$ & 0.629 \\
\hline $22: 5 \omega 6$ & 0.04 & 0.03 & 0.04 & 0.03 & 0.19 & 0.03 & 0.21 & 0.04 & 0.769 & $<0.001$ & 0.867 \\
\hline $22: 5 \omega 3$ & 0.01 & 0.01 & 0.04 & 0.03 & 0.29 & 0.06 & 0.25 & 0.04 & 0.935 & $<0.001$ & 0.414 \\
\hline $22: 6$ & $\begin{array}{c}0.50 \\
\text { Ratio }\end{array}$ & 0.07 & 0.27 & 0.08 & 0.51 & 0.11 & 0.60 & 0.08 & 0.472 & 0.065 & 0.088 \\
\hline $16: 0 / 16: 1$ & 2.93 & 0.13 & 3.50 & 0.22 & 4.16 & 0.14 & 4.56 & 0.20 & 0.013 & $<0.001$ & 0.634 \\
\hline $18: 0 / 18: 1$ & 0.04 & 0.005 & 0.04 & 0.005 & 0.06 & 0.006 & 0.07 & 0.006 & 0.654 & $<0.001$ & 0.810 \\
\hline Saturated / Unsaturated $\dagger$ & 0.42 & 0.02 & 0.45 & 0.01 & 0.70 & 0.02 & 0.72 & 0.02 & 0.142 & $<0.001$ & 0.813 \\
\hline
\end{tabular}

$+(12: 0+14: 0+15: 0+16: 0+17: 0+18: 0) /(16: 1+18: 1 \omega 9+18: 1 \omega 7+18: 2+18: 3 \omega 6+18: 3 \omega 3+20: 3 \omega 6+20: 4+20: 5+22: 4 \omega 6+22: 5 \omega 6+22: 5 \omega 3+22: 6)$

* Sidak post-hoc comparison $\mathrm{P}<0.05$

** Sidak post-hoc comparison $\mathrm{P}<0.01$

*** Sidak post-hoc comparison $\mathrm{P}<0.001$

Supplemental Table S3. Fatty acid composition of hepatic triglycerides.

Fatty acid composition of hepatic triglycerides from male $P d e 9 a^{+/+}$and $P d e 9 a^{-/-}$, fed either LFD or HFD. N = $10 P d e 9 a^{+/+}$LFD, $11 P d e 9 a^{-/-}$LFD, $13 P d e 9 a^{+/+}$ HFD, 16 Pde $9 a^{-/}$HFD. 